Pacific

Journal of

Mathematics

FUKUMOTO-FURUTA INVARIANTS OF PLUMBED HOMOLOGY 3-SPHERES

Nikolai SavelieV 


\title{
FUKUMOTO-FURUTA INVARIANTS OF PLUMBED HOMOLOGY 3-SPHERES
}

\author{
NikOLAI SAVELIEV
}

\begin{abstract}
Recent major progress in the study of the homology cobordism group of homology 3-spheres is related to the work of Fukumoto, Furuta, and Ue on their $w$-invariant. We identify the $w$-invariant with the $\bar{\mu}$-invariant of Neumann and Siebenmann for all plumbed homology spheres. This leads to proving that all Seifert fibered and some classes of plumbed homology spheres with nontrivial Rohlin invariant have infinite order in the homology cobordism group.
\end{abstract}

The homology cobordism group $\Theta^{3}$ of oriented integral homology 3 -spheres plays an outstanding role in the study of manifolds of high dimensions. Its most spectacular application is to the question of whether a closed topological manifold $M$ of dimension five or higher is a polyhedron. It is shown in [10] and [11] that every such $M$ is a polyhedron if and only if there exists a homology sphere with nontrivial Rohlin invariant which has second order in $\Theta^{3}$. It is not known if such a homology sphere exists. In fact, it is not known, at least to this author, whether $\Theta^{3}$ has any torsion at all or whether $\Theta^{3}$ modulo torsion is free abelian.

The group $\Theta^{3}$ has been studied successfully in the last two decades by the methods of gauge theory. The main focus of such studies has been on Seifert fibered homology spheres $\Sigma\left(a_{1}, \ldots, a_{n}\right)$ and other plumbed homology spheres. First, Fintushel and Stern $[3]$ have shown that certain manifolds $\Sigma\left(a_{1}, \ldots, a_{n}\right)$ have infinite order in $\Theta^{3}$. Then Furuta [8] has found an infinite collection of Seifert fibered homology spheres of infinite order all of which are linearly independent in $\Theta^{3}$, thus effectively establishing that $\Theta^{3}$ is an infinitely generated abelian group.

Gauge theoretical methods have also given a boost to the study of $\Theta^{3}$ via the $\bar{\mu}$-invariant of Neumann [12] and Siebenmann [18]. It has produced more examples of Seifert fibered homology spheres having infinite order in $\Theta^{3}$, see $[\mathbf{1 5}]$ and $[\mathbf{1 6}]$. Of particular importance in this study was the so called 10/8-condition on the signature of smooth closed spin 4-manifolds established by Furuta [9]. Recently, Fukumoto and Furuta [6] extended this result to spin $\mathrm{V}$-manifolds. For any integral homology sphere $\Sigma$, they introduced a family of invariants $w(\Sigma, X, c)$ of $\Sigma$ and a spin $\mathrm{V}$-manifold 
$(X, c)$ it bounds, and obtained some strong restrictions on the behavior of $w(\Sigma, X, c)$ whenever $\Sigma$ has finite order in $\Theta^{3}$.

In the special case of a Seifert fibered homology sphere $\Sigma=\Sigma\left(a_{1}, \ldots, a_{n}\right)$, Fukumoto, Furuta and Ue in [7] showed that $w(\Sigma, X, c)=-\bar{\mu}(\Sigma)$ for a specific choice of $(X, c)$. Based on this result, they proved that $\bar{\mu}$ is a homology cobordism invariant for all $\Sigma\left(a_{1}, \ldots, a_{n}\right)$ and their multiples provided one of the Seifert invariants $a_{i}$ is even, or else their number does not exceed six.

We develop a general theory of the $w$-invariant for plumbed homology spheres and plumbed V-manifolds. Plumbed V-manifolds comprise a rather large class of V-manifolds and include, in particular, all V-manifolds studied in [4] and [7] in connection with plumbed homology spheres. Every plumbed homology sphere bounds a spin plumbed V-manifold.

Theorem 1. Let $\Sigma$ be a plumbed homology sphere then $w(\Sigma, X, c)=-\bar{\mu}(\Sigma)$ for every spin plumbed $V$-manifold $(X, c)$ such that $\partial X=\Sigma$.

Using this theorem, we study homology cobordism properties of Seifert fibered and plumbed homology spheres. We notice that the results of [7] and [16] can be combined to remove the above mentioned restriction on Seifert fibered homology spheres of [7], and prove the following general result.

Theorem 2. Any Seifert fibered homology sphere $\Sigma\left(a_{1}, \ldots, a_{n}\right)$ with nonvanishing $\bar{\mu}$-invariant has infinite order in the homology cobordism group.

The modulo 2 reduction of the $\bar{\mu}$-invariant coincides with the Rohlin invariant. In particular, if the Rohlin invariant of $\Sigma\left(a_{1}, \ldots, a_{n}\right)$ is nontrivial then $\Sigma\left(a_{1}, \ldots, a_{n}\right)$ cannot have order two in $\Theta^{3}$. Thus if one is to look for an element in $\Theta^{3}$ solving positively the triangulation conjecture, all Seifert fibered homology spheres, as well as all homology spheres homology cobordant to them, should be excluded from consideration.

The methods of [6] and [7] are further applied to study homology cobordism properties of general plumbed homology spheres. It is not clear if these techniques are strong enough to prove that $\bar{\mu}$ is a homology cobordism invariant for all of them. However, we use them to construct an infinite family of plumbed homology spheres which are not Seifert fibered but for which the conclusion of Theorem 2 is true. This is a generalization of the results of $[4]$.

Theorem 3. Let $\Sigma$ be the splice of two Seifert fibered homology spheres $\Sigma\left(a_{1}, \ldots, a_{n}\right)$ and $\Sigma\left(\alpha_{1}, \ldots, \alpha_{m}\right)$ along fibers of degrees $a_{n}$ and $\alpha_{m}$, and let $p=a_{1} \ldots a_{n-1} \cdot \alpha_{1} \ldots \alpha_{m-1}-a_{n} \cdot \alpha_{m}$. Suppose that $\Sigma$ has finite order in the homology cobordism group. If $p \geq 0$ then $\bar{\mu}(\Sigma)=0$, and if $p<0$ then $\bar{\mu}(\Sigma)$ is either zero or one.

Note that such a $\Sigma$ is an algebraic link if and only if $p \leq 0$, and it is Seifert fibered if $p=0$. Thus the theorem does not provide us with a 
definite conclusion about $\bar{\mu}(\Sigma)$ precisely in the case when $\Sigma$ is a non-Seifert fibered algebraic link.

I am thankful to M. Furuta for useful discussions at Max-Planck-Institut für Mathematik in Bonn in 1998, and to Y. Fukumoto and M. Ue for sending me preprints [4], [5] and [7]. Discussions with W. Neumann and L. Siebenmann, as well as the referee's suggestions, were invaluable in bringing this paper into its final form.

\section{The $w$-invariants of Fukumoto and Furuta.}

In this section we review the $w$-invariants defined in [6] by using $\mathrm{V}$-manifolds. We formulate Fukumoto-Furuta's extension of the smooth 10/8-theorem to spin V-manifolds and show how it is related to certain homology cobordism properties of the $w$-invariants.

1.1. Closed V-manifolds. The general concept of V-manifold is described in [14]. In this paper, we need only a restricted class of $\mathrm{V}$-manifolds, namely compact $\mathrm{V}$-manifolds $X$ of dimension four with isolated singular points which may only occur in the interior of $X$. If $X$ is such a $\mathrm{V}$-manifold, each of its singular points has a neighborhood isomorphic to the cone over a lens space or some other linear spherical space form. The usual concepts of topology and differential geometry extend to $\mathrm{V}$-manifolds after defining everything in an equivariant setting. We will be interested in particular in a spin structure on $X$ and the (complex) index of the associated Dirac operator $\mathcal{D}(X)$. Fukumoto and Furuta proved in $[6]$ the following result about this index.

Theorem 4. Let $X$ be an oriented closed spin $V$-manifold with $b_{1}(X)=0$, and let $c$ be a spin structure on $X$. Then ind $\mathcal{D}(X)$ is even, and either ind $\mathcal{D}(X)=0$ or $-b_{-}(X)<$ ind $\mathcal{D}(X)<b_{+}(X)$.

Note that if $X$ is actually smooth, the statement of this theorem is equivalent to the 10/8-theorem of Furuta [9]. In this case, the index of Dirac operator is computed from the index theorem as

$$
\text { ind } \mathcal{D}(X)=-p_{1}(X) / 24=-\operatorname{sign}(X) / 8,
$$

which together with inequality $-b_{-}(X)<$ ind $\mathcal{D}(X)<b_{+}(X)$ of the theorem implies that $b_{2}(X) /|\operatorname{sign}(X)|>10 / 8$. In the special case of spin orbifolds whose only singularities are cones over lens spaces, Theorem 4 was also proved in $[\mathbf{1}]$.

1.2. Definition of $w$-invariants. Let $\Sigma$ be an oriented integral homology sphere, and let $X$ be a compact oriented spin $\mathrm{V}$-manifold with only isolated singularities, all in its interior, such that $b_{1}(X)=0$ and $\partial X=\Sigma$. Such a V-manifold always exists, moreover, it can be chosen to be smooth and 
simply connected. Let $c$ be a spin structure on $X$. For every triple $(\Sigma, X, c)$, the $w$-invariant is defined in $[\mathbf{6}]$ as

$$
w(\Sigma, X, c)=\text { ind } \mathcal{D}(X \cup Y)+\operatorname{sign}(Y) / 8,
$$

where $Y$ is any smooth spin 4-manifold with $\partial Y=-\Sigma$. Here, $\mathcal{D}(X \cup Y)$ is Dirac operator associated with the spin structure on $X \cup Y$ induced by $c$ and the spin structure on $Y$. Of course, if $X$ is smooth we simply get $w(\Sigma, X, c)=-\operatorname{sign}(X) / 8$.

The definition of $w(\Sigma, X, c)$ does not depend on the choice of $Y$. The reduction of $w(\Sigma, X, c)$ modulo 2 equals the Rohlin invariant of $\Sigma$ for any choice of $X$ and $c$. Moreover, $w(\Sigma, X, c)$ is additive with respect to connected sums of the triples $(\Sigma, X, c)$. All these statements are verified in [6]. Together with Theorem 4 they imply the following important property of the $w$-invariants.

Theorem 5. Let $\Sigma$ be an oriented integral homology sphere which has finite order in the homology cobordism group $\Theta^{3}$. Then, for any choice of $X$ and $c$, either $w(\Sigma, X, c)=0$ or

$$
-b_{-}(X)<w(\Sigma, X, c)<b_{+}(X) .
$$

For example, if $\Sigma$ happens to bound a spin $\mathrm{V}$-manifold $X$ such that $b_{+}(X) \leq 1, b_{-}(X) \leq 1$ and $w(\Sigma, X, c) \neq 0$, then $\Sigma$ cannot be of finite order in the homology cobordism group. The strength of Theorem 5 lies in the fact that many homology spheres $\Sigma$ do bound spin $\mathrm{V}$-manifolds $X$ with small values of $b_{ \pm}(X)$.

\section{Calculus of plumbing graphs.}

This section is devoted to 3- and 4-dimensional manifolds obtained by plumbing $D^{2}$-bundles over 2 -spheres, and to the $\bar{\mu}$-invariant of Neumann and Siebenmann.

2.1. Plumbed manifolds. A plumbing graph $\Gamma$ is a graph with no cycles, each of whose vertices $v_{i}$ carries an integer weight $e_{i}, i=1, \ldots, s$. Associate to each vertex $v_{i}$ the $D^{2}$-bundle $Y\left(e_{i}\right)$ over $S^{2}$ with the Euler number $e_{i}$. If the vertex $v_{i}$ has $d_{i}$ edges connected to it in the graph $\Gamma$, choose $d_{i}$ disjoint discs in the base of $Y\left(e_{i}\right)$ and call the disc bundle over the $j$-th disc $B_{i j}$, so that $B_{i j}=D^{2} \times D^{2}$. When two vertices, $v_{i}$ and $v_{k}$, are connected by an edge, identify $B_{i j}$ with $B_{k \ell}$ by exchanging the base and fiber coordinates. This pasting operation is called plumbing, and the resulting smooth 4-manifold $P(\Gamma)$ is said to be obtained by plumbing according to $\Gamma$. Its boundary $M(\Gamma)=\partial P(\Gamma)$ is usually referred to as a plumbed 3-manifold.

The 4-manifold $P(\Gamma)$ is simply connected. The group $H_{2}(P(\Gamma), \mathbb{Z})$ has a natural basis represented by the zero-sections of the plumbed bundles. We note that all these sections are embedded 2-spheres, and that they can be 
oriented in such a way that the intersection form of $P(\Gamma)$ will be given by the matrix $A(\Gamma)=\left(a_{i j}\right)_{i, j=1, \ldots, s}$ with the entries

$$
a_{i j}= \begin{cases}e_{i}, & \text { if } i=j \\ 1, & \text { if } i \text { is connected to } j \text { by an edge; } \\ 0, & \text { otherwise. }\end{cases}
$$

Let $M=\partial P(\Gamma)$ be a 3-dimensional manifold obtained by plumbing according to a graph $\Gamma$, then $M$ is an integral homology sphere if and only if the matrix $A(\Gamma)$ is unimodular, that is, $\operatorname{det} A(\Gamma)= \pm 1$.

Different plumbing graphs can describe the same 3-dimensional manifold. In fact, plumbing graphs $\Gamma_{1}$ and $\Gamma_{2}$ describe the same 3-manifold if and only if $\Gamma_{1}$ can be obtained from $\Gamma_{2}$ by a sequence of the moves shown in Figure 1 and their inverses. A proof of this statement can be found in the book [2] and also in the paper [18]. Each of the moves 1 and 2 will be called a blow down, and its inverse a blow up.

2.2. Seifert manifolds and lens spaces. Let $M$ be a 3 -manifold Seifert fibered over $S^{2}$ with Seifert invariants $\left(b ;\left(a_{1}, b_{1}\right), \ldots,\left(a_{n}, b_{n}\right)\right), a_{i} \geq 1$. It can be thought of as the boundary of the 4-manifold obtained by plumbing according to the star-shaped graph shown in Figure 2.

The integer weights $t_{i j}$ in this graph are found from continued fractions $a_{i} / b_{i}=\left[t_{i 1}, \ldots, t_{i m_{i}}\right]$ where $i=1, \ldots, n$ and we use the notation

$$
\left[t_{1}, \ldots, t_{k}\right]=t_{1}-\frac{1}{t_{2}-\frac{1}{\cdots-\frac{1}{t_{k}}}} .
$$

Different decompositions of $a_{i} / b_{i}$ into continued fractions lead to plumbing graphs describing the same 3-manifold $M$ but different 4-manifolds $P(\Gamma)$.

A Seifert manifold $M$ is an integral homology sphere if and only if the determinant of matrix $A(\Gamma)$ associated with the plumbing graph in Figure 2 is \pm 1 , which translates into the condition

$$
a_{1} \ldots a_{n} \cdot\left(-b+\sum_{i=1}^{n} b_{i} / a_{i}\right)= \pm 1 .
$$

We fix an orientation on $M$ by choosing +1 in (2). By considering (2) modulo $a_{i}$ we see that it determines $b_{i}$ modulo $a_{i}$ which is enough to determine the manifold $M$ completely. We use the notation $\Sigma\left(a_{1}, \ldots, a_{n}\right)$ for this manifold and call it a Seifert fibered homology sphere. 
1a. Delete a component of $\Gamma_{1}$ consisting of an isolated vertex with weight \pm 1 .

$1 \mathrm{~b}$.
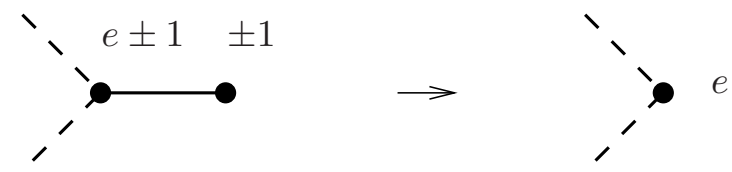

$1 c$
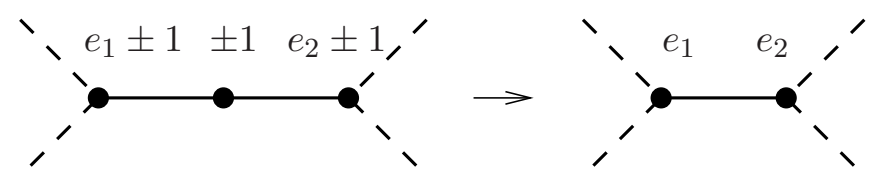

2 .
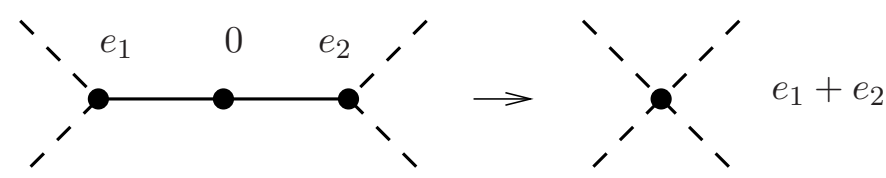

3.
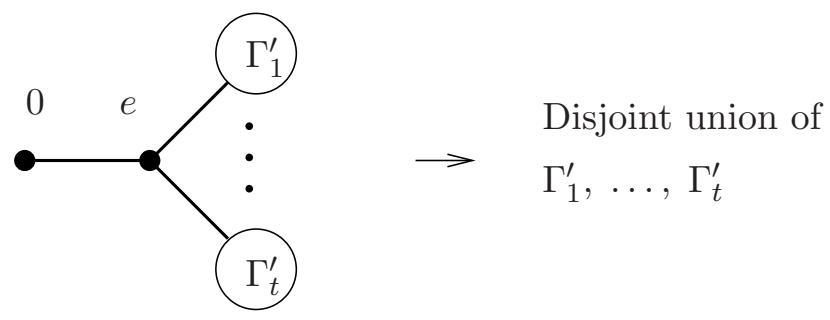

Figure 1.

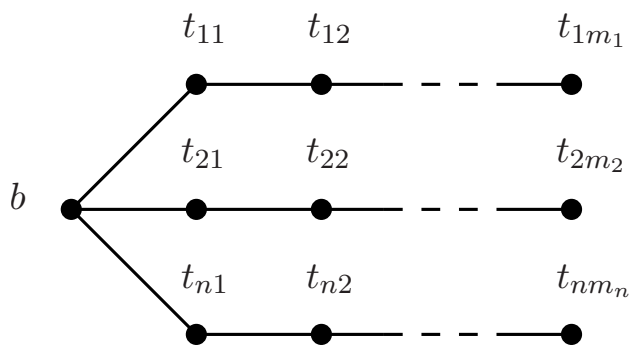

\section{Figure 2.}

In general, the order of the first homology of $M\left(b ;\left(a_{1}, b_{1}\right), \ldots,\left(a_{n}, b_{n}\right)\right)$ equals the absolute value of

$$
a_{1} \ldots a_{n} \cdot\left(-b+\sum_{i=1}^{n} b_{i} / a_{i}\right)
$$


unless the latter is equal to zero, in which case $H_{1}(M, \mathbb{Z})$ is infinite.

Lens spaces are a special case of Seifert fibered manifolds. Our orientation convention will be that a lens space $L(p, q)$ is obtained by $(-p / q)$ Dehn surgery on an unknot in $S^{3}$. Expanding $-p / q=\left[t_{1}, \ldots, t_{n}\right]$ into a continued fraction we get $L(p, q)$ as the boundary of the 4-manifold obtained by plumbing on the chain $\Gamma$ shown in Figure 3 . We will use notation $A(\Gamma)=\Delta\left(t_{1}, \ldots, t_{n}\right)$.

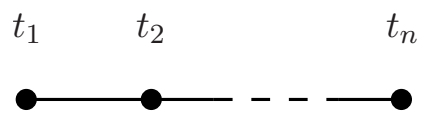

Figure 3.

One can notice that this plumbing graph also represents the lens space $L\left(p, q^{*}\right)$ with $-p / q^{*}=\left[t_{n}, \ldots, t_{1}\right]$ where $q q^{*}=1 \bmod p$. This reflects the fact that $L(p, q)$ and $L\left(p, q^{*}\right)$ are homeomorphic. Our convention is that lens spaces include only rational homology spheres. In particular, although $S^{3}$ and $S^{1} \times S^{2}$ can both be obtained by chain plumbing (see for instance Figure 4), we do not regard $S^{1} \times S^{2}$ as a lens space.

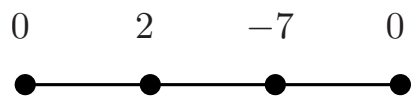

$S^{3}$

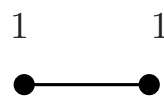

$S^{1} \times S^{2}$

\section{Figure 4.}

2.3. Intersection forms of plumbed 4-manifolds. There is a simple algorithm to diagonalize matrices of the form $A(\Gamma)$, see [2]. This algorithm is a graph realization of the usual Gauss diagonalization process for symmetric matrices. Let us allow trees weighted by arbitrary rational numbers. Given such a tree, pick a vertex and direct all its edges toward this vertex. Now, the tree can be simplified by performing operations of the two types shown in Figures 5 and 6 (we re-index vertices if necessary).

We end up with a finite collection of isolated points weighted by rational numbers $d_{1}, \ldots, d_{s}$. Then the diagonal matrix $D$ with the diagonal entries $d_{1}, \ldots, d_{s}$ is a diagonalization of $A(\Gamma)$, that is, $A(\Gamma)=U^{\top} D U$ with $\operatorname{det} U=$ \pm 1 . In particular, $\operatorname{det} A(\Gamma)=\operatorname{det} D$ and $\operatorname{sign} A(\Gamma)=\operatorname{sign} D$.

Example. For the tree $\Gamma$ in Figure 7 we have $\operatorname{det} A(\Gamma)=\operatorname{det} D=2 \cdot 3 \cdot$ $(1 / 6) \cdot(-2) \cdot(-1 / 2)=1$ and $\operatorname{sign} A(\Gamma)=\operatorname{sign} D=3-2=1$. 


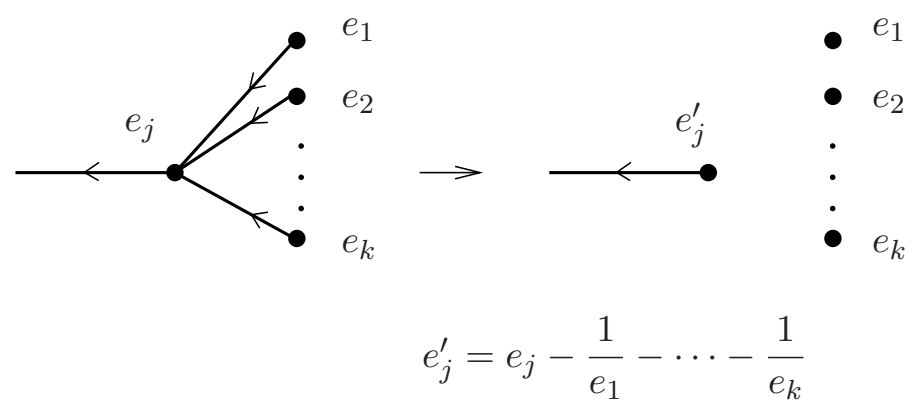

Figure 5.

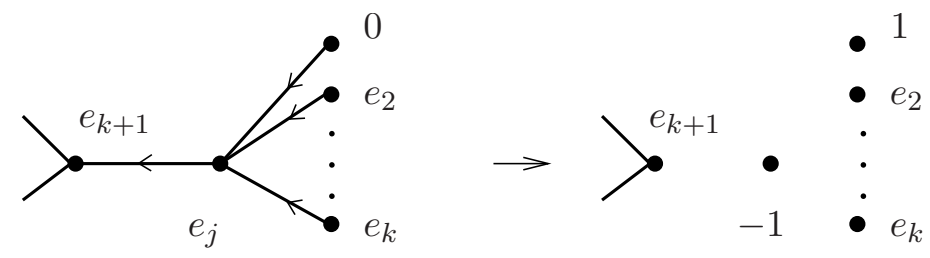

Figure 6.
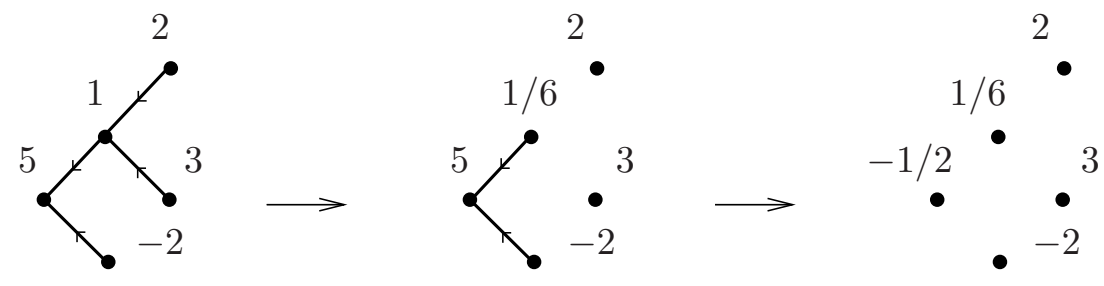

Figure 7.

Example. The diagonalization of the matrix $A(\Gamma)=\Delta\left(t_{1}, \ldots, t_{n}\right)$ corresponding to the tree in Figure 3 directed from right to left has entries

$$
\left[t_{n}\right],\left[t_{n-1}, t_{n}\right], \ldots,\left[t_{2}, \ldots, t_{n-1}, t_{n}\right],\left[t_{1}, \ldots, t_{n-1}, t_{n}\right] .
$$

The determinant of $\Delta\left(t_{1}, \ldots, t_{n}\right)$ equals (up to a sign) the numerator of the reduced fraction $\left[t_{1}, \ldots, t_{n-1}, t_{n}\right]$. We extend the concept of continued fraction by allowing division by zero in the sense of Figure 6 .

2.4. Graph links and splicing. By a link $(\Sigma, K)=\left(\Sigma, S_{1} \cup \cdots \cup S_{m}\right)$ we will mean a pair consisting of an oriented integral homology 3 -sphere $\Sigma$ and a collection $K$ of disjoint oriented knots $S_{1}, \ldots, S_{m}$ in $\Sigma$. Empty links are also allowed; they are just homology spheres. A link $\left(\Sigma\left(a_{1}, \ldots, a_{n}\right), S_{1} \cup \cdots \cup S_{m}\right)$ where the link components $S_{1}, \ldots, S_{m}$ are fibers in $\Sigma\left(a_{1}, \ldots, a_{n}\right)$ is called a Seifert link. Note that a general Seifert link may include singular fibers as well as some nonsingular fibers. 
Let $(\Sigma, K)$ and $\left(\Sigma^{\prime}, K^{\prime}\right)$ be links and choose components $S \subset K$ and $S^{\prime} \subset K^{\prime}$. Let $N(S)$ and $N\left(S^{\prime}\right)$ be tubular neighborhoods and $m, \ell \subset \partial N(S)$ and $m^{\prime}, \ell^{\prime} \subset \partial N\left(S^{\prime}\right)$ be standard meridians and longitudes. The manifold

$$
\Sigma^{\prime \prime}=(\Sigma \backslash \operatorname{int} N(S)) \cup\left(\Sigma^{\prime} \backslash \operatorname{int} N\left(S^{\prime}\right)\right)
$$

obtained by pasting along boundaries by matching $m$ to $\ell^{\prime}$ and $m^{\prime}$ to $\ell$, is an integral homology sphere. The link $\left(\Sigma^{\prime \prime},(K \backslash S) \cup\left(K^{\prime} \backslash S^{\prime}\right)\right)$ is called the splice of $(\Sigma, K)$ and $\left(\Sigma^{\prime}, K^{\prime}\right)$ along $S$ and $S^{\prime}$.

Any link which can be obtained from a finite number of Seifert links by splicing is called a graph link. Empty graph links are precisely plumbed, or graph, homology spheres, see [2].

Any graph link can be represented by a usual plumbing graph except some vertices of degree one in such a graph are left unweighted, and are drawn as arrowheads. An arrowhead vertex with the adjacent edge is referred to as an arrow. A plumbing graph $\Gamma$ with arrowheads represents a link $K$ in a 3-manifold $M$ as follows, see [2], pp. 134-135. Let $\bar{\Gamma}$ be $\Gamma$ with all the arrows deleted, and put $M=\partial P(\bar{\Gamma})$. Each arrowhead $v_{j}$ of $\Gamma$ is attached at some vertex $v_{i}$ of $\bar{\Gamma}$, and to this arrow we associate a fiber $S_{j}$ of the bundle $Y\left(e_{i}\right)$ used in plumbing. We take $S_{j}$ for different arrows $v_{j}$ to be disjoint, then $K$ is the union of these $S_{j}$.

Example. Figure 8 shows the Seifert link $\left(S^{3}, K\right)$ where $K$ is the right handed trefoil, corresponding to a regular fiber in a Seifert fibration of $S^{3}$.

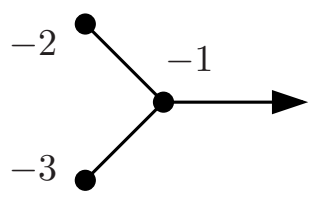

Figure 8 .

Example. Figure 9 shows the Seifert link $(\Sigma(2,3,7), K)$ with $K$ representing the singular fiber of degree 7 .

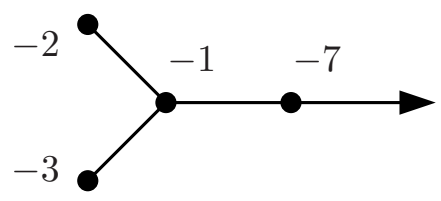

Figure 9. 
The splicing operation on graph links can be described in the language of plumbing graphs as follows. Suppose we are given two graph links represented by their plumbing diagrams $\Gamma$ and $\Gamma^{\prime}$ as shown in Figure 10 with arrows attached to vertices weighted by $e_{n}$ and $e_{m}^{\prime}$ respectively.

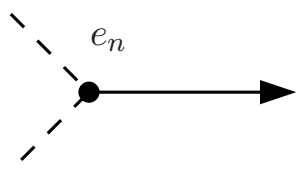

$\Gamma$

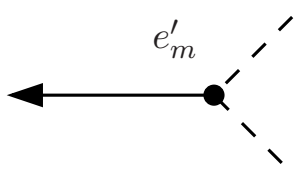

$\Gamma^{\prime}$

Figure 10.

The plumbing diagram for the spliced link is shown in Figure 11. Here, $a=\operatorname{det} A\left(\bar{\Gamma}_{0}\right) / \operatorname{det} A(\bar{\Gamma})$ where $\bar{\Gamma}$ is the plumbing graph $\Gamma$ with the arrow deleted, and $\bar{\Gamma}_{0}$ is the portion of $\bar{\Gamma}$ obtained by removing the $n$-th vertex weighted by $e_{n}$ and all its adjacent edges. The integer $a^{\prime}$ is obtained similarly from the graph $\Gamma^{\prime}$.

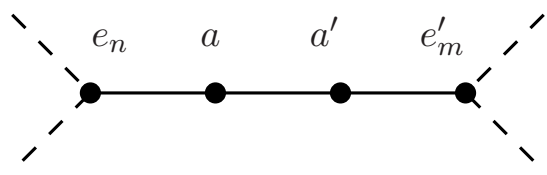

Figure 11.

Example. The diagrams in Figure 12 show plumbed homology spheres obtained by splicing two copies of $S^{3}$ along left- and right-handed trefoils (on the left), and by splicing $\Sigma(2,3,7)$ and $\Sigma(2,3,11)$ along singular fibers of degrees 7 and 11 (on the right). Both diagrams were simplified by using the usual rules.
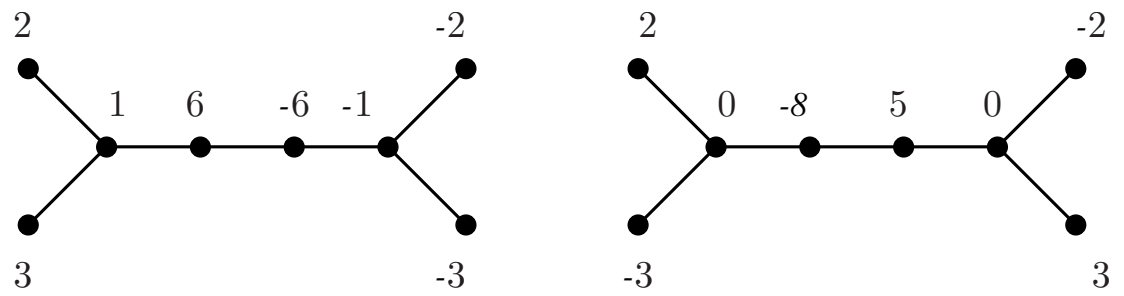

Figure 12. 
The above description of splicing in terms of plumbing graphs allows us to think of it as an operation on the corresponding plumbed 4-manifolds. This splicing operation in four dimensions can be described as follows. Let $W$ and $W^{\prime}$ be plumbed 4-manifolds with boundaries $\partial W=\Sigma$ and $\partial W^{\prime}=\Sigma^{\prime}$, and let $\Sigma^{\prime \prime}$ be the splice of $\Sigma$ and $\Sigma^{\prime}$ along knots $S$ and $S^{\prime}$. Represent $\Sigma$ as the union $\Sigma=X \cup(T \times I) \cup N(S)$ where the torus $T$ is the boundary of a tubular neighborhood $N(S)$ of $S, X$ is the knot exterior, and $I=[0,1]$. Let $\Sigma^{\prime}=X^{\prime} \cup(T \times I) \cup N\left(S^{\prime}\right)$ in a similar fashion. Then we can glue $W$ to $W^{\prime}$ along $T \times I$ to get a manifold with boundary $\Sigma^{\prime \prime} \cup S^{3}$, the $S^{3}$ component coming from gluing $N(S)$ with $N\left(S^{\prime}\right)$ by matching meridian to longitude and vice versa. Filling $S^{3}$ with 4 -dimensional ball, we get the same plumbed 4-manifold as from the operation on plumbing graphs.

2.5. The $\bar{\mu}$-invariant of plumbed homology spheres. For any plumbed homology sphere $\Sigma=\partial P(\Gamma)$, there exists a unique homology class $w \in$ $H_{2}(P(\Gamma), \mathbb{Z})$ satisfying the following two conditions. First, $w$ is characteristic, that is (dot represents intersection number)

$$
w \cdot x=x \cdot x \bmod 2 \text { for all } x \in H_{2}(P(\Gamma), \mathbb{Z}),
$$

and second, all the coordinates of $w$ are either 0 or 1 in the natural basis of $H_{2}(P(\Gamma), \mathbb{Z})$ represented by embedded 2 -spheres. We call $w$ the Wu class for $P(\Gamma)$. It is proved in [12] that the integer

$$
\bar{\mu}(\Sigma)=\frac{1}{8}(\operatorname{sign} P(\Gamma)-w \cdot w)
$$

only depends on $\Sigma$ and not on $\Gamma$. We call it the $\bar{\mu}$-invariant. One can easily see that condition (3) implies that for any two adjacent vertices in $\Gamma$ the corresponding coordinates of $w$ cannot both be equal to 1 . Therefore, the class $w$ can be chosen to be spherical, and $\bar{\mu}(\Sigma)=\mu(\Sigma)$ mod 2 where $\mu(\Sigma)$ is the Rohlin invariant.

Another description of the $\bar{\mu}$-invariant is as follows. In the natural basis of $H_{2}(P(\Gamma), \mathbb{Z})$, the defining property (3) translates into the linear system

$$
\sum_{j=1}^{s} a_{i j} w_{j}=a_{i i} \bmod 2, \quad i=1, \ldots, s .
$$

Due to the fact that $\operatorname{det} A(\Gamma)=1 \bmod 2$, this system has a unique solution $w=\left(w_{1}, \ldots, w_{s}\right)$ over $\mathbb{Z} / 2$. One can show that $w=\operatorname{diag} A(\Gamma)^{-1} \bmod 2$, see for example [17]. Hence $w_{i}$ can be computed as the mod 2 determinant of $A\left(\Gamma_{i}\right)$ where $\Gamma_{i}$ is obtained from $\Gamma$ by removing vertex $v_{i}$ and all its adjacent edges. The subset $\mathcal{S}$ of vertices $v_{i}$ of $\Gamma$ such that $w_{i}=1 \bmod 2$ is called the $\mathrm{Wu}$ set of $\Gamma$. The $\bar{\mu}$-invariant is then given by the formula

$$
\bar{\mu}(\Sigma)=\frac{1}{8}\left(\operatorname{sign} P(\Gamma)-\sum_{v_{i} \in \mathcal{S}} a_{i i}\right) .
$$


The $\bar{\mu}$-invariant is easy to compute. It is additive with respect to splicing, so one only needs to compute it for Seifert fibered homology spheres. Explicit formulas for $\bar{\mu}\left(\Sigma\left(a_{1}, \ldots, a_{n}\right)\right)$ can be found in [12].

All the above works as well for any plumbed $\mathbb{Z} / 2$-homology sphere $M$ thus defining $\bar{\mu}(M)$. It is no longer an integer although $8 \cdot \bar{\mu}(M)$ still is. For plumbed 3 -manifolds $M$ which are not $\mathbb{Z} / 2$-homology spheres, one can define an invariant $\bar{\mu}(M, c)$ which will depend on a spin structure $c$ on $M$, see $[\mathbf{1 2}]$ and $[\mathbf{1 7}]$.

\section{Plumbed V-cobordisms.}

In this section we take a different point of view on plumbed 4-manifolds and think of them as cobordisms between plumbed 3-manifolds and disjoint unions of lens spaces. We describe such cobordisms by decorated plumbing graphs.

3.1. Plumbed manifolds as V-cobordisms. Given a 4-manifold $P(\Gamma)$ obtained by plumbing on a graph $\Gamma$, let $\Gamma^{\prime}$ be a chain in $\Gamma$ of the form shown in Figure 3. Plumbing on $\Gamma^{\prime}$ yields a submanifold $P\left(\Gamma^{\prime}\right)$ of $P(\Gamma)$ with boundary a lens space $L(p, q)$. The closure of $P(\Gamma) \backslash P\left(\Gamma^{\prime}\right)$ is a smooth compact 4-manifold $X_{0}$ with oriented boundary $-L(p, q) \cup \partial P(\Gamma)$, which can be thought of as a cobordism between $L(p, q)$ and $\partial P(\Gamma)$. Starting with several chains $\Gamma_{k}^{\prime}$ in $\Gamma$, one can establish a cobordism between $\partial P(\Gamma)$ and a disjoint union $L$ of several lens spaces. The chains $\left\{\Gamma_{k}^{\prime}\right\}$ must be disjoint in the following sense: No two chains have a common vertex, and no edges of $\Gamma$ have one endpoint on one chain and the other endpoint on the other.

Such cobordisms will be called plumbed V-cobordisms. A plumbed Vcobordism $X_{0}$ is always a smooth manifold; the name $\mathrm{V}$-cobordism refers to the fact that each lens space on the boundary of $X_{0}$ can be capped off with a cone to make it into a $\mathrm{V}$-manifold $X$. The latter will be referred to as a plumbed V-manifold.

Plumbed V-cobordisms can be conveniently represented by decorated plumbing graphs. These are plumbing graphs $\Gamma$ with extra circles (not necessarily round) each of which encloses exactly one chain $\Gamma_{k}^{\prime}$. The above condition on the chains $\left\{\Gamma_{k}^{\prime}\right\}$ to be disjoint translates into the following conditions on the decorating circles: Any two planar 2-discs bounded by decorating circles are disjoint, and no edge of $\Gamma$ is intersected by more than one decorating circle.

Example. Consider the decorated plumbing graph in Figure 13. It describes a plumbed $\mathrm{V}$-cobordism $X_{0}$ between $L=L(2,-1) \cup L(2,1) \cup L(41,5)$ $\cup L(3,1) \cup L(3,-1)$ and $\Sigma$, where $\Sigma$ is the splice of $\Sigma(2,3,7)$ and $\Sigma(2,3,11)$ along the singular fibers of degrees 7 and 11, compare with Figure 12 . The only generators in the second homology of $X_{0}$ come from the vertices 


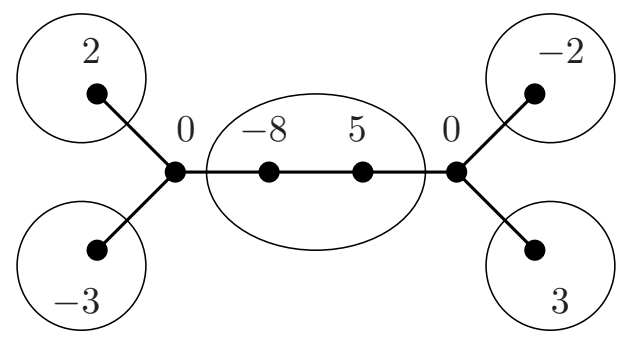

Figure 13.

outside all of the circles, that is the vertices weighted by 0 . Therefore, $H_{2}\left(X_{0}, \mathbb{Z}\right)=\mathbb{Z} \oplus \mathbb{Z}$.

3.2. Rational intersection forms of plumbed V-cobordisms. By the rational intersection form of a plumbed V-cobordism $X_{0}$ with $\partial X_{0}=-L \cup \Sigma$ we mean the cup-product pairing

$$
B: H^{2}\left(X_{0}, \mathbb{Q}\right) \otimes H^{2}\left(X_{0}, \mathbb{Q}\right) \rightarrow \mathbb{Q} .
$$

The form $B$ is nondegenerate over the rationals whenever $H_{1}(\Sigma, \mathbb{Q})=0$. The form $B$ is also referred to as the rational intersection form of the plumbed $\mathrm{V}$-manifold $X$, and by definition $\operatorname{sign} X=\operatorname{sign} X_{0}=\operatorname{sign} B$.

Let $X_{0}$ be represented by a decorated plumbing graph $\Gamma$ so that $P(\Gamma)=$ $\left(\bigcup P\left(\Gamma_{k}^{\prime}\right)\right) \cup_{L} X_{0}$ where $\Gamma_{k}^{\prime}$ are disjoint chains inside the decorating circles. Since $H_{1}(L, \mathbb{Q})=0$ we have a splitting

$$
A(\Gamma)=\bigoplus A\left(\Gamma_{k}^{\prime}\right) \oplus B
$$

In particular,

$$
\begin{gathered}
\operatorname{sign} A(\Gamma)=\sum \operatorname{sign} A\left(\Gamma_{k}^{\prime}\right)+\operatorname{sign} B, \quad \text { and } \\
\operatorname{det} A(\Gamma)=\prod \operatorname{det} A\left(\Gamma_{k}^{\prime}\right) \cdot \operatorname{det} B .
\end{gathered}
$$

Let $L=\bigcup L\left(p_{k}, q_{k}\right)$ and suppose that $\Sigma$ is an integral homology sphere. Then $\operatorname{det} A(\Gamma)= \pm 1$ and $\operatorname{det} B= \pm 1 / p_{1} \ldots p_{n}$.

In practical terms, the intersection form $B=\left(b_{i j}\right)$ can be calculated as follows. We call a vertex $v$ and a decorating circle adjacent if the circle intersects an edge one of whose endpoints is $v$.

The generators in the second homology of $X_{0}$ correspond to the vertices of $\Gamma$ outside all of the decorating circles. Given such a vertex $v_{i}$ weighted by an integer $e_{i}$, we have

$$
b_{i i}=e_{i}-\sum d_{k}, \quad d_{k}=\operatorname{det} A\left(\bar{\Gamma}_{k}^{\prime}\right) / \operatorname{det} A\left(\Gamma_{k}^{\prime}\right) .
$$

Here, the summation goes over all the indices $k$ such that $v_{i}$ is adjacent to the circle containing $\Gamma_{k}^{\prime}$. By $\bar{\Gamma}_{k}^{\prime}$ we denote the portion of $\Gamma_{k}^{\prime}$ obtained by 
removing the vertex inside $\Gamma_{k}^{\prime}$ adjacent to $v_{i}$ and deleting all its adjacent edges. We agree that the determinant of an empty graph is equal to 1.

For any two generating vertices $v_{i}$ and $v_{j}$ connected by an edge inside $\Gamma$ away from the decorating circles, $b_{i j}=1$. If these two vertices are adjacent to the same decorating circle enclosing a chain $\Gamma_{k}^{\prime}$, we have

$$
b_{i j}=\operatorname{det} A\left(\bar{\Gamma}_{k}^{\prime}\right) / \operatorname{det} A\left(\Gamma_{k}^{\prime}\right) \text {, }
$$

where $\bar{\Gamma}_{k}^{\prime}$ is the portion of $\Gamma_{k}^{\prime}$ obtained by removing all the vertices of $\Gamma_{k}^{\prime}$ belonging to the shortest chain connecting $v_{i}$ with $v_{j}$ and deleting all their adjacent edges. Note that the sign of $b_{i j}, i \neq j$, can be reversed by changing orientations of the generators $v_{i}$ or $v_{j}$. Since our plumbing graphs have no cycles, any choice of signs for $b_{i j}, i \neq j$, will do.

Example. The rational intersection form of the plumbed V-cobordism described by the decorated plumbing graph in Figure 13 is given by

$$
B=\left(\begin{array}{cc}
-11 / 246 & -1 / 41 \\
-1 / 41 & -7 / 246
\end{array}\right)
$$

Example. An important problem in calculations with plumbing graphs is to identify the extra lens space that arises from splicing, see Figure 14 where the lens space in question is circled.

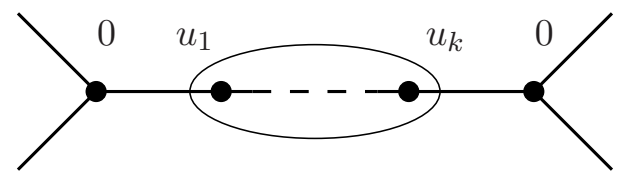

Figure 14.

This problem can be solved as follows. In the canonical meridian-longitude bases, the splicing is given by the formula

$$
\left(\begin{array}{c}
m \\
\ell
\end{array}\right)=\left(\begin{array}{ll}
0 & 1 \\
1 & 0
\end{array}\right)\left(\begin{array}{c}
m^{\prime} \\
\ell^{\prime}
\end{array}\right)
$$

To find the parameters of the lens space, one should re-write this gluing operation in the base-fiber bases. Suppose that we splice Seifert links $\left(\Sigma\left(a_{1}, \ldots, a_{n}\right), S\right)$ and $\left(\Sigma\left(\alpha_{1}, \ldots, \alpha_{m}\right), S^{\prime}\right)$ along Seifert fibers $S$ and $S^{\prime}$ of degrees $a_{n}$ and $\alpha_{m}$, respectively. Then

$$
\left(\begin{array}{c}
m \\
\ell
\end{array}\right)=\left(\begin{array}{cc}
a_{n} & b_{n} \\
-a_{1} \ldots a_{n-1} & d_{n}
\end{array}\right)\left(\begin{array}{l}
x \\
h
\end{array}\right),\left(\begin{array}{c}
m^{\prime} \\
\ell^{\prime}
\end{array}\right)=\left(\begin{array}{cc}
\alpha_{m} & \beta_{m} \\
-\alpha_{1} \ldots \alpha_{m-1} & \delta_{m}
\end{array}\right)\left(\begin{array}{l}
x^{\prime} \\
h^{\prime}
\end{array}\right)
$$


where

$$
d_{n}=a_{1} \ldots a_{n-1} \cdot \sum_{i=1}^{n-1} b_{i} / a_{i} \quad \text { and } \quad \delta_{m}=\alpha_{1} \ldots \alpha_{m-1} \cdot \sum_{j=1}^{m-1} \beta_{j} / \alpha_{j} .
$$

Therefore,

$$
\left(\begin{array}{l}
x \\
h
\end{array}\right)=\left(\begin{array}{cc}
q & -p^{*} \\
-p & -q^{*}
\end{array}\right)\left(\begin{array}{l}
x^{\prime} \\
h^{\prime}
\end{array}\right)
$$

where

$$
\begin{gathered}
p=a_{1} \ldots a_{n-1} \cdot \alpha_{1} \ldots \alpha_{m-1}-a_{n} \cdot \alpha_{m}, \\
q=-a_{1} \ldots a_{n-1} \cdot \alpha_{1} \ldots \alpha_{m-1} \cdot \sum_{i=1}^{n-1} b_{i} / a_{i}-b_{n} \cdot \alpha_{m}, \\
q^{*}=-a_{1} \ldots a_{n-1} \cdot \alpha_{1} \ldots \alpha_{m-1} \cdot \sum_{j=1}^{m-1} \beta_{j} / \alpha_{j}-\beta_{m} \cdot a_{n},
\end{gathered}
$$

and $p^{*}$ is determined from equation $p p^{*}+q q^{*}=1$ as

$$
p^{*}=-a_{1} \ldots a_{n-1} \cdot \alpha_{1} \ldots \alpha_{m-1} \sum_{i=1}^{n-1} b_{i} / a_{i} \sum_{j=1}^{m-1} \alpha_{j} / \beta_{j}+b_{n} \beta_{m} .
$$

The resulting lens space is $L(p, q)$ with the above parameters $p$ and $q$. Note that it only depends on the two spliced Seifert links and not on the rest of the manifold.

3.3. Spin structures on plumbed V-cobordisms. There exists a simple algorithm which determines whether a given plumbed $\mathrm{V}$-cobordism $X_{0}$ admits a spin structure. If such a spin structure exists it has to be unique because $H_{1}\left(X_{0}, \mathbb{Z}\right)=0$.

Given a V-cobordism $X_{0}$ represented by a decorated plumbing diagram $\Gamma$, consider the vertices outside all of the circles. They form a free basis in $H_{2}\left(X_{0}, \mathbb{Z}\right)$. Enumerate these vertices from 1 to $n$ and let $w_{i}=$ $\operatorname{det} A\left(\Gamma_{i}\right) \bmod 2$ where $\Gamma_{i}$ is obtained from $\Gamma$ by removing the $i$-th vertex and all adjacent edges, $i=1, \ldots, n$.

Proposition 6. The $V$-cobordism $X_{0}$ admits a spin structure if and only if $w_{i}=0 \bmod 2$ for all $i=1, \ldots, n$.

Proof. We work in the basis specified above. The $\mathrm{V}$-cobordism $X_{0}$ is spin if and only if its integral intersection matrix on $H_{2}\left(X_{0}, \mathbb{Z}\right)$ is even. The latter is the inverse of the rational intersection matrix $B$. The $i$-th diagonal element of $B^{-1}$ equals $\operatorname{det} B_{i} / \operatorname{det} B$, where $B_{i}$ is the minor obtained from $B$ by removing the $i$-th row and $i$-th column. 
To compute the determinants, let us introduce the following notation. The chains circled in the graph $\Gamma$ will be denoted by $\Gamma_{j}^{\prime}$. For every $j$, the lens space obtained by plumbing according to $\Gamma_{j}^{\prime}$ will be called $L\left(p_{j}, q_{j}\right)$, so that $p_{j}= \pm \operatorname{det} A\left(\Gamma_{j}^{\prime}\right)$. The determinant of $B$ can be calculated using the splitting (6) as

$$
\operatorname{det} B=\frac{\operatorname{det} A(\Gamma)}{\prod_{j} \operatorname{det} A\left(\Gamma_{j}^{\prime}\right)}= \pm \frac{1}{\prod_{j} p_{j}},
$$

where we take into account that $\operatorname{det} A(\Gamma)= \pm 1$ since $\Sigma$ is an integral homology sphere. Similarly,

$$
\operatorname{det} B_{i}=\frac{\operatorname{det} A\left(\Gamma_{i}\right)}{\prod_{j} \operatorname{det} A\left(\Gamma_{j}^{\prime}\right)}
$$

Therefore, $w_{i}=\operatorname{det} B_{i} / \operatorname{det} B=\operatorname{det} A\left(\Gamma_{i}\right) \bmod 2$, and the result follows.

Example. The plumbed V-cobordism $X_{0}$ shown in Figure 13 is spin. This can be seen in two different ways. One is to find its rational intersection form $B$ and its inverse,

$$
B^{-1}=\left(\begin{array}{cc}
-42 & 36 \\
36 & -66
\end{array}\right)
$$

and note that $B^{-1}$ is even. The other is to apply Proposition 6 as follows. Enumerate the vertices generating $H_{2}\left(X_{0}, \mathbb{Z}\right)$ from left to right. Removing vertex number 1 together with its adjacent edges leaves us with the graph $\Gamma_{1}$ shown in Figure 15. The determinant of $A\left(\Gamma_{1}\right)$ is the product of the determinants of the connected components of $\Gamma_{1}$. The one vertex component in the upper left corner has even determinant, therefore, $w_{1}=0 \bmod 2$. Similarly, removing vertex number 2 , it can be seen that $w_{2}=0 \bmod 2$, so that the $\mathrm{V}$-cobordism $X_{0}$ is spin.

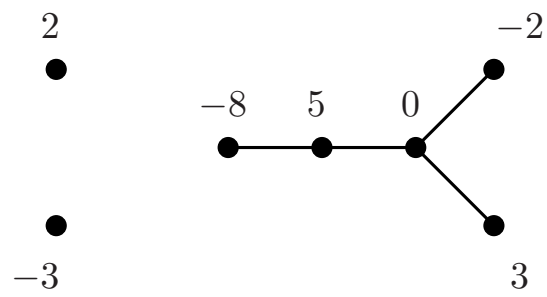

\section{Figure 15.}

Let $X_{0}$ be a $\mathrm{V}$-cobordism represented by a decorated plumbing graph $\Gamma$, and $v_{j}$ a vertex outside all of the decorating circles. We say that $v_{j}$ is in the Wu set of $X_{0}$ if $w_{j}=1 \bmod 2$. Thus $X_{0}$ is spin if and only if its Wu set is empty. Proposition 6 implies that the $\mathrm{Wu}$ set of $X_{0}$ is calculated by the 
same rules as the $\mathrm{Wu}$ set of (undecorated) plumbing graph $\Gamma$, see Section 2.5. Therefore, one can simply say that the Wu set of $X_{0}$ consists of the vertices of $\Gamma$ which belong to the Wu set of $\Gamma$ and sit outside all of the decorating circles. This observation implies the following result.

Corollary. Every plumbed homology sphere bounds a spin plumbed V-cobordism.

Proof. Let $\Sigma$ be an integral homology 3 -sphere obtained by plumbing according to a graph $\Gamma$. Since the $\mathrm{Wu}$ set of $\Gamma$ contains no adjacent vertices, we can draw one circle around each vertex $v_{i}$ with $w_{i}=1$, and the resulting circles will be disjoint. An extra precaution should be taken not to get a zero-weighted vertex inside any of the circles. This can be easily avoided by blowing up the graph $\Gamma$ at the corresponding vertices. The graph $\Gamma$ with so drawn circles represents a spin plumbed V-cobordism with boundary $\Sigma$.

3.4. Spin V-cobordisms for Seifert fibered homology spheres. Let $\Sigma\left(a_{1}, \ldots, a_{n}\right)$ be a Seifert fibered homology sphere such that one of the invariants, say $a_{1}$, is even. Using the freedom allowed by Equation (2), one can choose Seifert invariants $\left(b ;\left(a_{1}, b_{1}\right), \ldots,\left(a_{n}, b_{n}\right)\right)$ so that $b_{1}$ is odd, $b$ is even and all $b_{i}$ with $i=2, \ldots, n$, are even. Each $a_{i} / b_{i}$ admits then a continued fraction expansion $\left[t_{i 1}, \ldots, t_{i m_{i}}\right]$ with all $t_{i j}$ even, so that the manifold obtained by plumbing on the graph in Figure 2 is spin. We can make it into a spin V-cobordism $X_{0}$ by decorating as shown in Figure 16 .

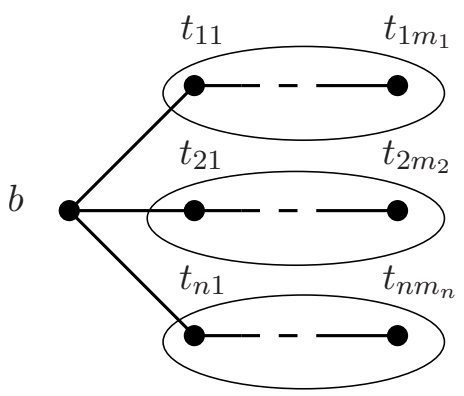

Figure 16.

We see immediately that $H_{2}\left(X_{0}, \mathbb{Z}\right)=\mathbb{Z}$. The rational intersection form of $X_{0}$ is $b-\sum b_{i} / a_{i}=-1 / a_{1} \ldots a_{n}$, so that it is negative definite, with $b_{+}=0$ and $b_{-}=1$.

Let $\Sigma\left(a_{1}, \ldots, a_{n}\right)$ be a Seifert fibered homology sphere with all invariants $a_{i}$ odd. For any choice of Seifert invariants $\left(0 ;\left(a_{1}, b_{1}\right), \ldots,\left(a_{n}, b_{n}\right)\right)$, consider the standard plumbing graph $\Gamma$ as in Figure 2 (we choose $b=0$ for the sake of simplicity). The central vertex of $\Gamma$ weighted by 0 is in the $\mathrm{Wu}$ set for any choice of Seifert invariants. Blow $\Gamma$ up $(n-1)$ times to get the graph 
shown in Figure 17. The 0-framed vertices adjacent to the central vertex are not in the Wu set of the blown up graph, so if we decorate it as shown in Figure 17 we will get a spin plumbed V-cobordism $X_{0}$.

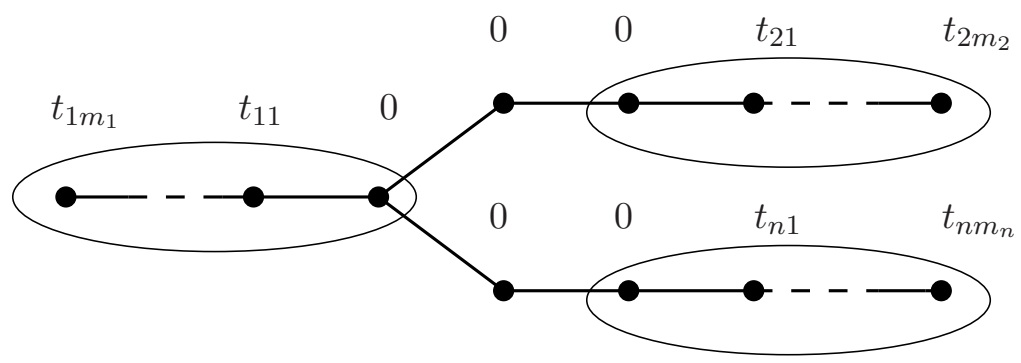

Figure 17.

Proposition 7. The signature of the rational intersection form $B$ of spin plumbed $V$-cobordism $X_{0}$ shown in Figure 17 equals $\sum_{i=1}^{n} \operatorname{sign}\left(a_{i} / b_{i}\right)-1$.

Proof. Let us consider the decorated plumbing graph in Figure 18 and the associated rational intersection form $B^{\prime}$. The (undecorated) plumbing graphs in Figures 17 and 18 represent the same plumbed homology sphere, and one can check that their intersection forms have equal signatures. Splitting these forms over the rationals, we get

$$
\sum_{i=1}^{n} \operatorname{sign} \Delta\left(0, t_{i 1}, \ldots, t_{i m_{i}}\right)+\operatorname{sign} B=\sum_{i=1}^{n} \operatorname{sign} \Delta\left(t_{i 1}, \ldots, t_{i m_{i}}\right)+\operatorname{sign} B^{\prime} .
$$

Since $\operatorname{sign} \Delta\left(t_{i 1}, \ldots, t_{i m_{i}}\right)=\operatorname{sign} \Delta\left(0, t_{i 1}, \ldots, t_{i m_{i}}\right)+\operatorname{sign}\left(a_{i} / b_{i}\right)$ by the diagonalization algorithm, and $\operatorname{sign} B^{\prime}=-1$, we are finished.

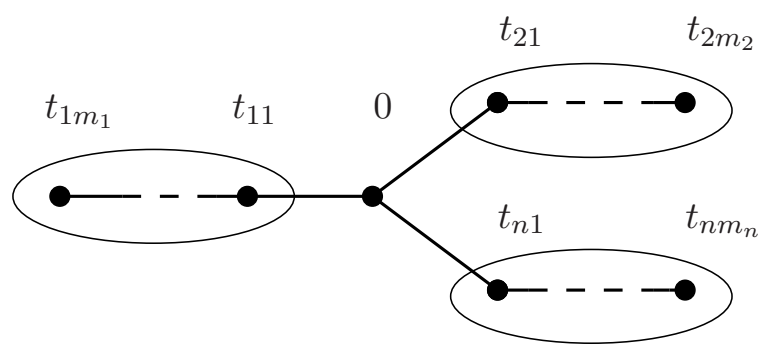

Figure 18. 


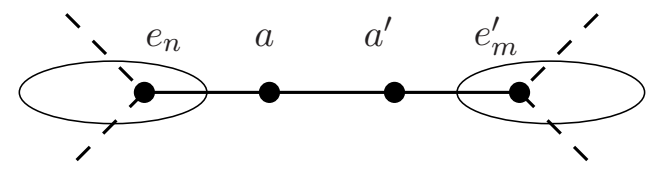

Figure 19.

3.5. Splicing plumbed V-cobordisms. Given two plumbed V-cobordisms described by decorated plumbing graphs, we splice them as (undecorated) plumbing graphs as shown in Figure 11, and keep the decorations on both components unchanged. Thus in the gluing region the spliced graph may look as shown in Figure 19.

Note that the two new vertices, framed in Figure 19 by $a$ and $a^{\prime}$, do not belong to the $\mathrm{Wu}$ set of the spliced graph because by removing either of them together with the two adjacent edges, we split the graph in two connected components with determinants 0 and \pm 1 . The latter follows easily from the formulas for $a$ and $a^{\prime}$ given in Section 2.4. Therefore, the splice of two spin plumbed V-cobordisms is again a spin plumbed V-cobordism.

\section{The $w$-invariants for spin plumbed V-cobordisms.}

In this section, we use an explicit formula for the $w$-invariant given in [7] to prove that, for any spin plumbed $\mathrm{V}$-manifold $(X, c)$ with boundary $\Sigma$, we have $w(\Sigma, X, c)=-\bar{\mu}(\Sigma)$. This implies in particular that, given a plumbed integral homology sphere $\Sigma$, the integer $w(\Sigma, X, c)$ is independent of the choice of a spin plumbed $\mathrm{V}$-manifold $(X, c)$.

4.1. A formula for the $w$-invariants. Let $X$ be a compact oriented spin V-manifold with $b_{1}(X)=0, \partial X=\Sigma$, and with only isolated singularities which are the cones over lens spaces, $L\left(p_{i}, q_{i}\right)$. Let $Y$ be a smooth spin 4manifold with $\partial Y=-\Sigma$ then ind $\mathcal{D}(X \cup Y)$ and therefore $w(\Sigma, X, c)$ can be calculated explicitly by applying the $\mathrm{V}$-index theorem. Such a calculation in [7] provides us with the formula

$$
w(\Sigma, X, c)=-\frac{1}{8}\left(\operatorname{sign}(X)+\sum \sigma\left(q_{i}, p_{i}, \varepsilon_{i}\right)\right),
$$

where sign $(X)$ stands for the signature of the rational intersection form of $X$, and functions $\sigma(q, p, \varepsilon)$ for any relatively prime integers $p$ and $q$ and $\varepsilon= \pm 1$ are defined by the formula

$$
\sigma(q, p, \varepsilon)=\frac{1}{p} \sum_{k=1}^{|p|-1}\left(\cot \left(\frac{\pi k}{p}\right) \cot \left(\frac{\pi k q}{p}\right)+2 \varepsilon^{k} \csc \left(\frac{\pi k}{p}\right) \csc \left(\frac{\pi k q}{p}\right)\right) .
$$

For a spin plumbed $\mathrm{V}$-manifold $X$, the parameter $\varepsilon$ can be calculated as follows. Let $X$ be described by a decorated plumbing graph $\Gamma$ and let $L(p, q)$ be the lens space obtained by plumbing on a chain $\Gamma^{\prime}$ inside one of 
the decorating circles. Suppose that the chain looks like the one in Figure 3, with $-p / q=\left[t_{1}, \ldots, t_{n}\right]$. The $\mathrm{Wu}$ set of the graph $\Gamma$ is uniquely determined; in particular, we know which of the vertices of $\Gamma^{\prime}$ belong to this Wu set. We set $\varepsilon=1$ if the vertex of $\Gamma^{\prime}$ weighted by $t_{1}$ is in the Wu set, and $\varepsilon=-1$ otherwise. In particular, if $p$ is odd, $\varepsilon=(-1)^{q-1}$.

The paper [7] gives us the formula $\varepsilon=(-1)^{w(m)-1}$ where $L(p, q)$ is thought of as $(-p / q)$-surgery on an unknot $K$ in $S^{3}$ with meridian $m$, and with the spin structure given by $w \in H^{1}\left(S^{3} \backslash K, \mathbb{Z} / 2\right)$. The latter obviously agrees with the spin structure described by plumbing on a chain, so the consistency of two formulas for $\varepsilon$ will follow from the next lemma.

Lemma 8. The intersection of the $W u$ set of $\Gamma$ with the set of vertices of $\Gamma^{\prime}$ is a Wu set of $\Gamma^{\prime}$.

Proof. If $p$ is odd then $L(p, q)$ is a $\mathbb{Z} / 2$-homology sphere. Therefore, the intersection form $A(\Gamma)$ of $P(\Gamma)$ splits over $\mathbb{Z} / 2$ in two summands, one of which is $A\left(\Gamma^{\prime}\right)$, and hence the Wu set also splits. If $p$ is even, blow up $\Gamma$ near $\Gamma^{\prime}$ as shown in Figure 20.

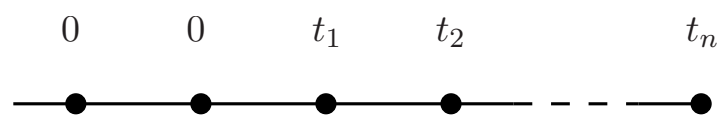

\section{Figure 20.}

Let $\Gamma^{\prime \prime}$ be the chain with vertices framed by $0, t_{1}, \ldots, t_{n}$. Since $\operatorname{det} A\left(\Gamma^{\prime \prime}\right)$ is odd we can apply the argument above and conclude that the restriction of the $\mathrm{Wu}$ set of $\Gamma$ on $\Gamma^{\prime \prime}$ is a $\mathrm{Wu}$ set of $\Gamma^{\prime \prime}$. To complete the proof we only need to check that the restriction of the $\mathrm{Wu}$ set of $\Gamma^{\prime \prime}$ on $\Gamma^{\prime}$ is a $\mathrm{Wu}$ set of $\Gamma^{\prime}$, which is immediate.

4.2. The $\sigma$-function. The function $\sigma(q, p, \varepsilon)$ has the following properties, proved in [7], which in practice allow to calculate it without actually referring to its definition:

(1) $\sigma(q+c p, p, \varepsilon)=\sigma\left(q, p,(-1)^{c} \varepsilon\right)$.

(2) $\sigma(-q, p, \varepsilon)=\sigma(q,-p, \varepsilon)=-\sigma(q, p, \varepsilon)$.

(3) If $p+q$ is odd then $\sigma(p, q,-1)+\sigma(q, p,-1)=\operatorname{sign}(p q)$, in particular, $\sigma(1, p,-1)=-\operatorname{sign} p$ for any even $p$.

(4) For any $p, q$ such that $|p|>|q|$ and $p+q$ is odd, there exists a continued fraction decomposition $p / q=\left[t_{1}, \ldots, t_{n}\right]$ with all $t_{i}$ even and $\left|t_{i}\right| \geq 2$. Then $\sigma(q, p,-1)=-\sum_{i=1}^{n} \operatorname{sign} t_{i}$.

We will show that in fact $\sigma(q, p, \varepsilon)$ is nothing else but the $\bar{\mu}$-invariant of $L(p, q)$. To be more precise, if $p$ is odd then $L(p, q)$ is a $\mathbb{Z} / 2$-homology sphere, so the $\bar{\mu}$-invariant is defined as in Section 2.5 and only depends on $L(p, q)$ and not on the choice of a plumbing graph. The only difference is that 
$\bar{\mu}(L(p, q))$ is not an integer although $8 \cdot \bar{\mu}(L(p, q))$ still is. If $p$ is even, there are two different $\mathrm{Wu}$ classes because of the fact that $H^{1}(L(p, q), \mathbb{Z} / 2)=\mathbb{Z} / 2$. Suppose that $L(p, q)$ is obtained by plumbing on a chain $\Gamma^{\prime}$ like the one shown in Figure 3 so that $-p / q=\left[t_{1}, \ldots, t_{n}\right]$. Since $q= \pm\left[t_{2}, \ldots, t_{n}\right]$ is odd, the $\mathrm{Wu}$ set of $A\left(\Gamma^{\prime}\right)$ is uniquely determined by whether the first vertex of $\Gamma^{\prime}$, weighted by $t_{1}$, is in the $\mathrm{Wu}$ set. We say that $v=1$ if it is in the $\mathrm{Wu}$ set and $v=0$ otherwise. Fixing $v$ allows to define the $\bar{\mu}$-invariants $\bar{\mu}(L(p, q), v)$ as before.

Proposition 9. If $p$ is odd then $\sigma\left(q, p,(-1)^{q-1}\right)=8 \bar{\mu}(L(p, q))$. If $p$ is even, $\sigma\left(q, p,(-1)^{v-1}\right)=8 \bar{\mu}(L(p, q), v)$.

Proof. First we note that we may assume without loss of generality that $|p|>|q|$. Suppose that $p$ is odd and $q$ is even. We find a continued fraction decomposition $p / q=\left[t_{1}, \ldots, t_{n}\right]$ as in Property (4) and evaluate $\sigma(q, p,-1)=-\sum_{i=1}^{n} \operatorname{sign} t_{i}=-\operatorname{sign} \Delta\left(t_{1}, \ldots, t_{n}\right)$ (the latter equality is true because all $\left|t_{i}\right|$ are greater than or equal to 2$)$. Since the $\mathrm{Wu}$ set is empty, $8 \bar{\mu}(L(p, q))=-\operatorname{sign} \Delta\left(t_{1}, \ldots, t_{n}\right)$.

If both $p$ and $q$ are odd, we use Property (1) to get $\sigma(q, p, 1)=\sigma(q-$ $p, p,-1)$ with even $q-p$. By the previous argument, $\sigma(q-p, p,-1)=$ $8 \bar{\mu}(L(p, q-p))$. Let $-p /(q-p)=\left[t_{1}, \ldots, t_{n}\right]$ with all $t_{i}$ even, then $-p / q=$ $\left[0,1, t_{1}, \ldots, t_{n}\right]$ with $\mathrm{Wu}$ set consisting of just one vertex weighted by 0 . Since sign $\Delta\left(t_{1}, \ldots, t_{n}\right)=\operatorname{sign} \Delta\left(0,1, t_{1}, \ldots, t_{n}\right)$ by the diagonalization algorithm, we see that $\bar{\mu}(L(p, q-p))=\bar{\mu}(L(p, q))$, which completes the proof in this case.

If $p$ is even, one should consider two separate cases, $v=0$ and $v=1$. The first is treated as the case of odd $p$ and even $q$, with all even $t_{i}$ which provides for $v=0$. The case $v=1$ is completely similar to the case of odd $p$ and $q$, if one keeps in mind that adding two vertices framed by 0 and 1 does not change the $\mathrm{Wu}$ set of $\Delta\left(t_{1}, \ldots, t_{n}\right)$.

It should be mentioned that Proposition 9 might give easy proofs of various number-theoretical properties of $\sigma(q, p, \varepsilon)$ proved in [7].

4.3. Proof of Theorem 1. Let $X$ be a spin plumbed V-manifold described by a decorated plumbing graph $\Gamma$ with chains $\Gamma_{i}^{\prime}, i=1, \ldots, n$, inside the decorating circles, so that $\partial P(\Gamma)=\Sigma$ and $\partial P\left(\Gamma_{i}^{\prime}\right)=L\left(p_{i}, q_{i}\right)$. Let $w_{i}$ be the Wu classes of $A\left(\Gamma_{i}^{\prime}\right), i=1, \ldots, n$, and $w$ the Wu class of $A(\Gamma)$. Since $X$ is 
spin, $w=w_{1}+\cdots+w_{n}$. Then

$$
\begin{array}{rlrl}
w(\Sigma, X, c) & =-\left(\operatorname{sign} X_{0}+\sum \sigma\left(q_{i}, p_{i}, \varepsilon_{i}\right)\right) / 8, & & \text { by formula }(7), \\
& =-\left(\operatorname{sign} X_{0}+\sum\left(\operatorname{sign} P\left(\Gamma_{i}^{\prime}\right)-w_{i} \cdot w_{i}\right)\right) / 8, & & \text { by Proposition } 9, \\
& =-\left(\operatorname{sign} X_{0}+\sum \operatorname{sign} P\left(\Gamma_{i}^{\prime}\right)-w \cdot w\right) / 8, & & \text { since } X_{0} \text { is spin, } \\
& =-(\operatorname{sign} P(\Gamma)-w \cdot w) / 8, & & \text { by formula }(6), \\
& =-\bar{\mu}(\Sigma) . &
\end{array}
$$

In particular we see that $w(\Sigma, X, c)$ is independent of the choice of $(X, c)$ as long as we stay in the class of spin plumbed V-manifolds.

\section{Homology cobordism properties of the $\bar{\mu}$-invariant.}

Using Theorem 1, we can reformulate Theorem 5 of Fukumoto and Furuta $[6]$ as follows.

Theorem 10. Let $\Sigma$ be a plumbed homology sphere bounding a spin plumbed $V$-manifold $X$. If $\Sigma$ has finite order in the homology cobordism group then either $\bar{\mu}(\Sigma)=0$ or $-b_{-}(X)<-\bar{\mu}(\Sigma)<b_{+}(X)$.

In this section, we apply this result to study the homology cobordism properties of Seifert fibered and plumbed homology spheres. We generalize results obtained in this direction in $[4],[6]$, and $[7]$.

5.1. Proof of Theorem 2. Let $\Sigma=\Sigma\left(a_{1}, \ldots, a_{n}\right)$ be a Seifert fibered homology sphere with one of the $a_{i}$ even. Let $X$ be the plumbed $\mathrm{V}$-manifold shown in Figure 16. It is spin and it has $b_{-}(X)=1$ and $b_{+}(X)=0$. By Theorem 10, if $\Sigma$ has finite order in the homology cobordism group, $\bar{\mu}(\Sigma)$ must vanish.

Let $\Sigma=\Sigma\left(a_{1}, \ldots, a_{n}\right)$ be a Seifert fibered homology sphere with all the $a_{i}$ odd, and suppose it has finite order in the homology cobordism group. Choose invariants $b_{i}$ so that all $a_{i} / b_{i}$ but one are positive. Then the plumbed spin V-manifold $X$ shown in Figure 17 has $b_{+}(X)+b_{-}(X)=n-1$ and $b_{+}(X)-b_{-}(X)=n-3$, see Proposition 7. Therefore, $b_{+}(X)=n-2$ and $b_{-}(X)=1$, and Theorem 10 implies that

$$
0 \leq-\bar{\mu}(\Sigma)<n-2 .
$$

Now choose a different set of invariants $\left(a_{i}, b_{i}\right)$ so that all $a_{i} / b_{i}$ but one are negative. The above argument with the $\mathrm{V}$-manifold as in Figure 17 shows that

$$
1-n<-\bar{\mu}(\Sigma) \leq 0 .
$$

These inequalities together prove that $\bar{\mu}(\Sigma)$ vanishes. 
There is another way to prove the theorem for Seifert fibered homology spheres $\Sigma=\Sigma\left(a_{1}, \ldots, a_{n}\right)$ with all $a_{i}$ odd. It uses inequality (8) and the result of $[\mathbf{1 6}]$ which says that if an algebraic link has finite order in the homology cobordism group, its $\bar{\mu}$-invariant is nonnegative. Since $\Sigma\left(a_{1}, \ldots, a_{n}\right)$ is an algebraic link, we conclude that $-\bar{\mu}(\Sigma) \leq 0$, which completes the proof.

5.2. Proof of Theorem 3. Let us denote Seifert invariants of $\Sigma\left(a_{1}, \ldots, a_{n}\right)$ and $\Sigma\left(\alpha_{1}, \ldots, \alpha_{m}\right)$ respectively by $\left(a_{i}, b_{i}\right)$ and $\left(\alpha_{j}, \beta_{j}\right)$. The splicing construction provides us with the plumbing graph $\Gamma$ shown in Figure 21, which we decorate as indicated. One may assume without loss of generality that the $\mathrm{V}$-manifold $X$ we obtain is spin: If all the $a_{i}$ and $\alpha_{j}$ are odd this is automatic; if some of them are even certain precautions should be made in choosing $b_{i}$ and $\beta_{j}$.

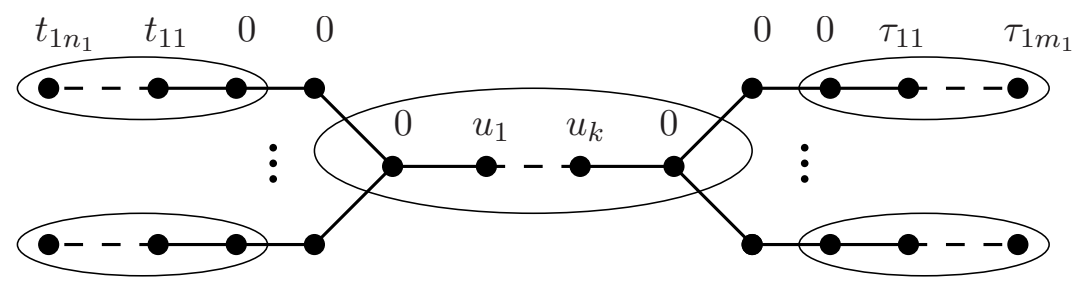

Figure 21.

We use here the notations $a_{i} / b_{i}=\left[t_{i 1}, \ldots, t_{i n_{i}}\right], \alpha_{j} / \beta_{j}=\left[\tau_{j 1}, \ldots, \tau_{j m_{j}}\right]$, $p / q=\left[u_{1}, \ldots, u_{k}\right]$ and $p / q^{*}=\left[u_{k}, \ldots, u_{1}\right]$, where the parameters $p, q$ and $q^{*}$ were computed in Section 3.2.

Let us denote the rational intersection form of $X$ by $B$, and consider another plumbed $\mathrm{V}$-manifold $X^{\prime}$ given by the plumbing graph $\Gamma^{\prime}$ decorated as shown in Figure 22.

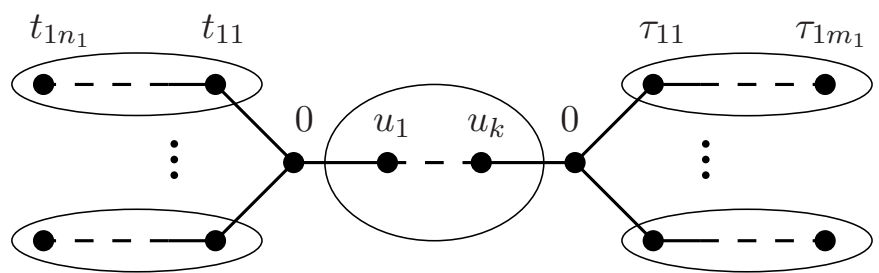

Figure 22.

Let $B^{\prime}$ be the rational intersection matrix of $X^{\prime}$. One can easily see that the matrices $A(\Gamma)$ and $A\left(\Gamma^{\prime}\right)$ have the same signature. Decomposing these 
two matrices over the rationals provides us with the identity

$$
\begin{gathered}
\operatorname{sign} B+\sum_{i=1}^{n-1} \operatorname{sign} \Delta\left(0, t_{i 1}, \ldots, t_{i n_{i}}\right)+\sum_{j=1}^{m-1} \operatorname{sign} \Delta\left(0, \tau_{j 1}, \ldots, \tau_{j m_{j}}\right) \\
+\operatorname{sign} \Delta\left(0, u_{1}, \ldots, u_{k}, 0\right)=\operatorname{sign} B^{\prime}+\sum_{i=1}^{n-1} \operatorname{sign} \Delta\left(t_{i 1}, \ldots, t_{i n_{i}}\right) \\
+\sum_{j=1}^{m-1} \operatorname{sign} \Delta\left(\tau_{j 1}, \ldots, \tau_{j m_{j}}\right)+\operatorname{sign} \Delta\left(u_{1}, \ldots, u_{k}\right) .
\end{gathered}
$$

By the diagonalization algorithm,

$$
\operatorname{sign} \Delta\left(0, t_{i 1}, \ldots, t_{i n_{i}}\right)-\operatorname{sign} \Delta\left(t_{i 1}, \ldots, t_{i n_{i}}\right)=-\operatorname{sign}\left(a_{i} / b_{i}\right),
$$

and similarly for $\alpha_{j} / \beta_{j}$. The difference

$$
\operatorname{sign} \Delta\left(0, u_{1}, \ldots, u_{k}, 0\right)-\operatorname{sign} \Delta\left(u_{1}, \ldots, u_{k}\right)
$$

is computed as follows. Decompose the matrix $\Delta\left(0, u_{1}, \ldots, u_{k}, 0\right)$ over the rationals into the direct sum of $\Delta\left(u_{1}, \ldots, u_{k}\right)$ and the rational intersection matrix $U$ of the decorated plumbing graph shown in Figure 23.

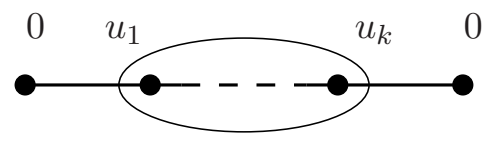

\section{Figure 23.}

Then $\operatorname{sign} \Delta\left(0, u_{1}, \ldots, u_{k}, 0\right)-\operatorname{sign} \Delta\left(u_{1}, \ldots, u_{k}\right)=\operatorname{sign} U$. The two-bytwo matrices $B^{\prime}$ and $U$ are computed by the usual rules as

$$
B^{\prime}=\left(\begin{array}{cc}
-\sum b_{i} / a_{i}-q / p & -1 / p \\
-1 / p & -\sum \beta_{j} / \alpha_{j}-q^{*} / p
\end{array}\right), \quad U=\left(\begin{array}{cc}
-q / p & -1 / p \\
-1 / p & -q^{*} / p
\end{array}\right),
$$

and the resulting formula for $\operatorname{sign} B$ is

$$
\operatorname{sign} B=\operatorname{sign} B^{\prime}-\operatorname{sign} U+\sum_{i=1}^{n-1} \operatorname{sign}\left(a_{i} / b_{i}\right)+\sum_{j=1}^{m-1} \operatorname{sign}\left(\alpha_{j} / \beta_{j}\right) .
$$

After simplification using explicit formulas from Section 3.2, we obtain

$$
B^{\prime}=\left(\begin{array}{cc}
\alpha_{m} /\left(a_{1} \ldots a_{n-1} \cdot p\right) & -1 / p \\
-1 / p & a_{n} /\left(\alpha_{1} \ldots \alpha_{m-1} \cdot p\right)
\end{array}\right)
$$

and $\operatorname{det} B^{\prime}=-1 /\left(a_{1} \ldots a_{n-1} \cdot \alpha_{1} \ldots \alpha_{m-1} \cdot p\right)$. Thus $\operatorname{sign} B^{\prime}=0$ if $p>0$, and sign $B^{\prime}=-2$ if $p<0$ (the latter could be seen alternatively from the fact that, in the case when $p$ is negative, $\Sigma$ is an algebraic link and therefore 
bounds a negative definite plumbed manifold). Concerning the matrix $U$, we compute

$$
\begin{aligned}
& \operatorname{det} U=-p^{*} / p \\
& =\frac{a_{1} \ldots a_{n-1} \cdot \alpha_{1} \ldots \alpha_{m-1} \sum b_{i} / a_{i} \sum \alpha_{j} / \beta_{j}-a_{n} \cdot \alpha_{m}\left(b_{n} / a_{n}\right)\left(\beta_{m} / \alpha_{m}\right)}{a_{1} \ldots a_{n-1} \cdot \alpha_{1} \ldots \alpha_{m-1}-a_{n} \cdot \alpha_{m}},
\end{aligned}
$$

where the summation extends over $i=1, \ldots, n-1$ and $j=1, \ldots, m-$ 1. Replacing $\sum b_{i} / a_{i}$ by $\sum b_{i} / a_{i}+k$ at the expense of changing $b_{n} / a_{n}$ to $b_{n} / a_{n}-k$, and doing the same with the other set of invariants, we see that $\operatorname{det} U$ is positive at least for large $|k|$. If $k$ is large positive, sign $U=-2$; if $k$ is negative and large in absolute value, sign $U=2$.

Suppose that $\Sigma$ has finite order in the homology cobordism group, and that $p$ is positive. Then sign $B^{\prime}=0$. If we choose $b_{i} / a_{i}, i=1, \ldots, n-1$, and $\alpha_{j} / \beta_{j}, j=1, \ldots, m-1$, to be large positive then sign $B=n+m-4$. Since the rank of $B$ is $n+m-2$, we conclude that $b_{+}=n+m-3, b_{-}=1$, and

$$
-1<-\bar{\mu}(\Sigma)<n+m-3 .
$$

If we choose $b_{i} / a_{i}, i=1, \ldots, n-1$, and $\alpha_{j} / \beta_{j}, j=1, \ldots, m-1$, to be negative and of large absolute value then $\operatorname{sign} B=-n-m+4$ so that $b_{+}=1, b_{-}=n+m-3$, and

$$
3-n-m<-\bar{\mu}(\Sigma)<1 .
$$

These two sets of inequalities on $\bar{\mu}(\Sigma)$ imply that $\bar{\mu}(\Sigma)=0$.

Suppose now that $p$ is negative then $\operatorname{sign} B^{\prime}=-2$ and arguing as above we get inequalities

$$
-2<-\bar{\mu}(\Sigma)<n+m-4 \text { and } 2-n-m<-\bar{\mu}(\Sigma) \leq 0,
$$

which imply that $\bar{\mu}(\Sigma)$ is either zero or one. This completes the proof.

\section{References}

[1] D. Acosta, A Furuta-like inequality for spin orbifolds and the minimal genus problem, Topology Appl., 114 (2001), 91-106, CMP 1830 326, Zbl 0983.57027.

[2] D. Eisenbud and W. Neumann, Three-Dimensional Link Theory and Invariants of Plane Curve Singularities, Princeton Univ. Press, Princeton, 1985, MR 87g:57007, Zbl 0628.57002.

[3] R. Fintushel and R. Stern, Pseudofree orbifolds, Ann. Math., 122 (1985), 335-364, MR 87a:57027, Zbl 0602.57013.

[4] Y. Fukumoto, On an invariant of plumbed homology 3-spheres, J. Math. Kyoto Univ., 40 (2000), 379-388, MR 2001m:57032, Zbl 0973.57015.

[5] _ Plumbed homology 3-spheres bounding acyclic 4-manifolds, J. Math. Kyoto Univ., 40 (2000), 731-751, MR 2002g:57065. 
[6] Y. Fukumoto and M. Furuta, Homology 3-spheres bounding acyclic 4-manifolds, Math. Res. Lett., 7 (2000), 757-766, MR 2001m:57063, Zbl 0971.57026.

[7] Y. Fukumoto, M. Furuta and M. Ue, W-invariants and Neumann-Siebenmann invariants for Seifert homology 3-spheres, Topology Appl., 116(3) (2001), 333-369, CMP 1857670.

[8] M. Furuta, Homology cobordism group of homology 3-spheres, Inv. Math., 100 (1990), 339-355, MR 91c:57039, Zbl 0716.55008.

[9] _ Monopole equation and the 11/8 conjecture, Math. Res. Lett., 8(3) (2001), 279-291, CMP 1839478.

[10] D. Galewski and R. Stern, Classification of simplicial triangulations of topological manifolds, Ann. Math., 111 (1980), 1-34, MR 81f:57012, Zbl 0441.57017.

[11] T. Matumoto, Variétés Simpliciales d'Homologie et Variétés Topologiques Métrisables, These de doctorat d'etat, Université de Paris XI - Centre d'Orsay, Octobre 1976.

[12] W. Neumann, An invariant of plumbed homology spheres, in 'Topology Symposium', Siegen 1979, 125-144, Lecture Notes in Math., 788, Springer, Berlin, 1980, MR 82j:57033, Zbl 0436.57002.

[13] W. Neumann and J. Wahl, Casson invariant of links of singularities, Comm. Math. Helv., 65 (1990), 58-78, MR 91c:57022, Zbl 0704.57007.

[14] I. Satake, The Gauss-Bonnet theorem for V-manifolds, J. Math. Soc. Japan, 9 (1957), 464-492., MR 20 \#2022, Zbl 0080.37403.

[15] N. Saveliev, Dehn surgery along torus knots, Topology Appl., 83 (1998), 193-202, MR 99a:57030, Zbl 0928.57015.

[16] N Notes on homology cobordisms of plumbed homology 3-spheres, Proc. Amer. Math. Soc., 126 (1998), 2819-2825, MR 98k:57036, Zbl 0903.57011.

[17] _ A surgery formula for the $\bar{\mu}$-invariant, Topology Appl., 106 (2000), 91-102, MR 2001g:57025, Zbl 0960.57010.

[18] L. Siebenmann, On vanishing of the Rohlin invariant and nonfinitely amphicheiral homology 3-spheres, in 'Topology Symposium', Siegen 1979, 172-222, Lecture Notes in Math., 788, Springer, Berlin, 1980, MR 81k:57011, Zbl 0444.57003.

Received November 7, 2000 and revised June 6, 2001. This research was supported in part by NSF Grants DMS-9896376 and DMS-0071480 and Max-Planck-Institut für Mathematik in Bonn.

Department of Mathematics

UNIVERSITY OF MiAMI

P.O.Box 249085

Coral Gables, FL 33124-4250

E-mail address: saveliev@math.miami.edu 